\title{
DETECCIÓN DE NECESIDADES Y COMPETENCIA EMPRENDEDORA EN TIEMPOS DE PANDEMIA: ADAPTACIÓN A LA FORMACIÓN EN DANZA ONLINE ${ }^{l}$
}

\author{
DETECTION OF NEEDS AND ENTREPRENEURIAL COMPETENCE \\ IN TIMES OF PANDEMIC: \\ ONLINE ADAPTATION TO DANCE TRAINING
}

\begin{abstract}
Natalia Ollora-Triana ${ }^{a}$, Tamara de la Torre-Cruz ${ }^{b},{ }^{a}{ }^{a}$ del Camino EscolarLlamazares ${ }^{b}$, Cristina Di Giusto-Valle ${ }^{b},{ }^{a} \cdot{ }^{a}$ Isabel Luis-Rico ${ }^{b}$, M. $^{a}$ del Carmen Palmero-Cámara ${ }^{b}$ y Juan Alfredo Jiménez Eguizábal ${ }^{b}$
\end{abstract}

Fechas de recepción y aceptación: 9 de junio de 2021 y 14 de julio de 2021

DOI: https://doi.org/10.46583/edetania_2021.60.902

Resumen: Durante el confinamiento mundial provocado por la COVID-19, la práctica de actividades rítmicas y bailadas en tiempo de ocio se ha incrementado, manteniéndose la demanda hasta la actualidad. El confinamiento provoca que estas actividades se hayan realizado de forma no presencial y las continuas restricciones obligan a adaptar la actividad a un formato mixto. El objetivo de esta investigación es conocer qué aspectos de la competencia emprendedora han implementado estos profesionales para afrontar con éxito este crecimiento en la demanda. El sector se adapta a un nuevo formato de práctica nunca planteado y supera la situación de crisis mediante el uso de la competencia emprendedora, innovando y manteniendo la ocupación empresarial. La investigación es cualitativa y la metodología de análisis pretende contribuir al conocimiento a partir de la teoría fundamentada construida tras analizar sistemáticamente las entrevistas realizadas a emprendedores de escuelas de danza. Las entrevistas se estructuran en torno a seis bloques: aspectos sociodemográficos; aspectos empresariales;

a Facultad de Educación. Universidad de la Rioja.

* Correspondencia: Universidad de la Rioja. Facultad de Educación. Edificio de Rectorado. Avenida de La Paz, 93-103. 26006 Logroño. La Rioja. España.

E-mail: naollot@unirioja.es

${ }^{\text {b }}$ Facultad de Educación. Universidad de Burgos.

${ }^{1}$ Proyecto PID2019-104408GB-I00/AEI/10.13039/501100011033. Formación del Potencial Emprendedor. Generación de un Modelo Educativo de Identidad Emprendedora. Plan Estatal 2017-2020 Generación Conocimiento - Proyectos I+D+i (2020-2023). 
espíritu empresarial; espíritu emprendedor; competencias personales y atributos, y conocimientos y aspectos vinculados a la actividad como práctica de ocio. Para el análisis se utiliza el software ATLAS TI Cloud. La discusión de resultados concluye que los emprendedores han encontrado la motivación en el compromiso social a partir de su sensibilidad personal para enfrentar la realidad con decisión. Crean un nuevo producto sopesando riesgos e identificando la nueva demanda como una oportunidad. Igualmente, han emprendido proyectos ambiciosos dando como resultado una sociedad más equitativa y una nueva adhesión a la práctica de esta actividad.

Palabras clave: competencia emprendedora, ocio, escuelas de danza, pandemia COVID-19.

Abstract: During the global confinement caused by COVID-19, the practice of rhythmic and dance activities in leisure time has increased, maintaining the demand until today. Confinement means that these activities have been carried out in a non-face-to-face way and the continuous restrictions make it necessary to adapt the activity to a mixed format. The objective of this research is to know what aspects of entrepreneurial competence have been implemented by these professionals to successfully face this growth in demand. The sector adapts to a new practice format never planned and overcomes the crisis situation through the use of entrepreneurial competence, innovating and maintaining the business occupation. The research is qualitative and the analysis methodology aims to contribute to the knowledge based on the Grounded Theory built after systematically analyzing the interviews carried out with dance school entrepreneurs. The interviews are structured around six blocks: socio-demographic aspects; business aspects; entrepreneurship; entrepreneurial spirit; personal skills and attributes and, knowledge and aspects related to the activity as a leisure practice. The ATLAS TI Cloud software is used for the analysis. The discussion of results concludes that entrepreneurs have found motivation in social commitment based on their personal sensitivity to face reality decisively. They create a new product by weighing risks and identifying the new demand as an opportunity. Likewise, they have undertaken ambitious projects resulting in a more equitable society and a new adherence to the practice of this activity.

Keywords: entrepreneurial competence, leisure, dance schools, COVID-19 pandemic.

\section{INTRODUCCIÓN}

La pandemia provocada por la COVID-19 dejó a la población mundial confinada en cuestión de horas. El decreto de estado de alarma ha llevado a los sectores profesionales a una diferenciación entre los considerados esenciales, que debían mantener su actividad y el resto de las profesiones no consideradas esenciales (RDL 463/2020, del 14 de marzo). La evolución de la economía y el mercado de trabajo, tanto nacional como mundial, se ha visto afectada por el 
impacto de una crisis sin precedentes. En este entorno de elevada incertidumbre para todos los sectores de la población, todavía se desconoce la totalidad de sus consecuencias, que se verán reflejadas a corto y medio plazo (Social, 2021).

Las circunstancias de confinamiento, que se han ido sucediendo a lo largo de la situación de pandemia, se caracterizan por el aislamiento y la restricción de movimiento, tan necesario para el ser humano (Andreu-Cabrera, 2020). La reclusión, la soledad o la convivencia continuada en el mismo entorno generan consecuencias negativas en los hábitos saludables y descompensaciones en diferentes patologías relacionadas no solo con la salud mental y física (Márquez, 2020), sino también con la salud social, por lo que desde el inicio de la pandemia una de las recomendaciones de los diferentes especialistas en salud es la realización de actividad física para ayudar a contrarrestar los posibles efectos secundarios físicos y mentales de una situación temporal nunca vivida por la población (Hammami, Harrabi, Mohr y Krustrup, 2020). Los profesionales recomiendan llevar a cabo rutinas diarias entre las que debe realizarse una disciplina física para ayudar a reducir los efectos del confinamiento (Andreu-Cabrera, 2020; Lara-Aparicio, Mayorga-Vega y López-Fernández, 2021). Adicionalmente, se ha descubierto la importancia de la participación en actividades físicas de disfrute y que se perciban motivadoras para producir beneficios en la salud de la población (Lakes et al., 2016).

De esta forma, estas actividades no consideradas esenciales y los profesionales del sector se transforman en necesarios para la población, situando a las empresas que desarrollan actividades físicas en un lugar privilegiado de oportunidad para la innovación y la adaptación. Recuérdese, en este sentido, que el informe del mercado de trabajo estatal (SEPE, 2020) advierte de que unas de las ocupaciones con mejores perspectivas para los emprendedores son, entre otras, las asociadas a los profesionales de actividades recreativas y de entretenimiento y las que desarrollan diferentes actividades físicas, por estar vinculadas fundamentalmente al ocio y a la mejora de la calidad de vida de las personas y el cuidado personal.

Dentro de las actividades más escogidas por la población para la práctica en el hogar están las actividades rítmicas y bailadas, lo que genera un incremento en el número de consumidores desde el inicio de la pandemia (Dalmases, 2020; Encalada, 2020). Las experiencias relacionadas con estas actividades se 
han convertido en una vía de escape, desahogo físico y emocional, por lo que en pocos meses se ha visto proliferar las clases de danza en línea propuestas desde diferentes plataformas y escuelas (Burzynska, Taylor y Knecht, 2017; Fancourt y Finn, 2019; Bohn y Hogue, 2021). Se han escogido como práctica de ocio por diferentes grupos sociales con el objetivo de abordar el aislamiento y la inactividad con menos consecuencias para la salud, revelando una mejora en la apreciación corporal y en la conexión social (Hansen, Main y Hartling, 2021; Trevino et al., 2021). Los programas basados en la danza refuerzan los trastornos del estado de ánimo provocados por la ansiedad, la depresión, la excitabilidad y los ataques de pánico, además de paliar los posibles daños cardiovasculares y pulmonares derivados de la inactividad y el estado continuo de reposo (Re, 2021). Su práctica, tanto en formato en línea como presencial, combina aspectos físicos y psicosociales, promueve la expresión de sentimientos y estados emocionales en las personas, mejora la autoestima y la autoconfianza, además de aliviar el estrés (Capel, Cruvinel, Mendoça y Celeno, 2018). Incluso se han empleado para reparar la función motora, pulmonar, el estado psicológico y otros aspectos con pacientes que se recuperan de la COVID-19, por lo que se ha encontrado en las actividades físicas y motoras apoyadas en la danza y el ritmo una mejora significativa para la recuperación de secuelas, como los estados de ansiedad, depresión e insomnio (Ding et al., 2021).

Las empresas vinculadas al sector han sido conscientes de que esta actividad se había convertido en una necesidad para toda la población, y de la misma forma que algunas industrias no esenciales han modificado su proceso productivo para elaborar productos necesarios (Haeffele, Hobson y Storr, 2020), el sector de las escuelas de danza a partir de la adaptación ha ofrecido el mejor servicio a pesar de las circunstancias. De forma coyuntural y llevadas por la particularidad de demanda creciente de su actividad, muchas de estas empresas han puesto su empeño y esfuerzo al servicio del emprendimiento social, ofreciendo su producto para dar respuesta a una necesidad social, explotando la oportunidad de llevar su actividad a toda una población antes no afiliada a su práctica mediante la gestión y organización innovadora (Espínola y Torres, 2020). Algunos emprendedores ofrecieron la posibilidad desde su plataforma de empresa de poder recibir clases de danza con diferentes maestros de distintos lugares del mundo, siguiendo el modelo de pago por servicio (Espínola y Torres, 2020). Así, durante el primer confinamiento prevalecieron las clases 
virtuales, ofrecidas por estos emprendedores de forma libre y gratuita a través de diferentes plataformas (Capasso et al., 2020; Bohn y Hogue, 2021), obedeciendo a la necesidad social creada por la situación y teniendo en cuenta la incertidumbre temporal.

La velocidad con la que estos empresarios convirtieron su servicio en un bien social ha sido decisiva en la práctica de la danza y su actual adhesión por la población como práctica de ocio (García-Parada y Aguado, 2020), convirtiéndose en una de las actividades en auge desarrolladas y con mejores perspectivas dentro de la educación no reglada (SEPE, 2020). La base en la respuesta del sector se encuentra en su competencia emprendedora, que les ha permitido llevar a cabo las acciones concretas desde el inicio de la nueva situación. Las actitudes y habilidades, junto con el conocimiento, son elementos competenciales cuya movilización es necesaria para el desempeño de las funciones del emprendedor (Alda-Varas, Villardón-Gallego y Elexpuru-Albizuri, 2012), además de la aptitud como un componente del emprendedor que se origina de la conducta y tiene carácter permanente, estable, innato y no aprendido (De la Torre, Luis, Palmero, Escolar y Jiménez, 2019). Algunas de las dimensiones que se vinculan con las habilidades y destrezas de la persona emprendedora son la creatividad, las relaciones sociales y la sensibilidad (Alda-Varas, VillardónGallego y Elexpuru-Albizuri, 2012). La dimensión referida a los conocimientos está integrada por los conocimientos generales para el aprendizaje, los vinculados a la actividad y los profesionales. Las actitudes y los valores que poseen se caracterizan por la asunción de riesgos, autoestima o confianza en uno mismo, control percibido interno, necesidad de logro, orientación a la innovación, planificación, iniciativa, compromiso personal e identificación de oportunidades (Enciso-Congote, 2010; Alda-Varas, Villardón-Gallego y Elexpuru-Albizuri, 2012; De la Torre et al., 2019). Por último, la competencia emprendedora se asocia a la aptitud (De la Torre et al., 2019), que implica la capacidad de asumir responsabilidades y de comunicación, autonomía, mejora del aprendizaje y de las actuaciones personales, liderazgo, pensamiento crítico, trabajo en equipo, toma de decisiones (individuales y colectivas), utilización de la tecnología y, por último, capacidad de negociación (Enciso-Congote, 2010; Damas, 2012; De la Torre et al., 2019).

Otras competencias asociadas al emprendedor son la tolerancia al fracaso (Enciso-Congote, 2010), la actitud mental positiva, la gestión y optimización 
de recursos y compromiso (De la Torre et al., 2019) y la inteligencia emocional (Aragón y Baixauli, 2010).

Por otro lado, el informe sobre la situación del emprendimiento en España ante la crisis originada por la COVID-19 (Fuentes-Fuentes y Neira-Gómez, 2021) refleja que un $96 \%$ de las personas encuestadas ve oportunidades en este momento de dificultad, manifestándose la identificación de oportunidades como otro de los componentes del emprendedor (Alda-Varas, Villardón-Gallego y Elexpuru-Albizuri, 2012).

Estos recursos competenciales han sido necesarios en este momento de crisis nunca vivida y sitúa a las figuras del emprendedor y empresario como una de las palancas de cambio para la recuperación del crecimiento y la creación de empleo para la prosperidad futura (Jiménez-Zarco, 2012; Fuentes-Fuentes y Neira-Gómez, 2021). Tanto es así que, siendo la danza una actividad eminentemente práctica y de índole motora, se han encontrado maneras creativas para mantener y fomentar la conexión de los usuarios, estando físicamente separados de forma obligada (Haeffele, Hobson y Storr, 2020), hasta lograr que los consumidores se sientan apoyados y escuchados por los trabajadores de las escuelas (Jaramillo, 2015).

Es en épocas de crisis cuando surgen nuevos emprendedores o las empresas ya creadas modifican su negocio para adaptarse, recuperarse económicamente y crear empleo (Jiménez-Zarco, 2012) a través de la creatividad y la innovación, pudiendo convertirse en un dinamizador de la economía. Esta figura ha visto los problemas como oportunidades y con capacidad se ha adaptado a las circunstancias y necesidades cambiantes (Nicolás y Rubio, 2020).

Nuestro interés investigador se centra en el desarrollo del modelo de competencia emprendedora que permita establecer qué conocimientos, habilidades, valores, actitudes y aptitudes han sido necesarios en los empresarios al frente de las escuelas de danza para lograr el reto de mantener una actividad no esencial que ha requerido de adaptaciones según las características conformantes. Es decir, las competencias necesarias y suficientes para que, en la actual situación de crisis, estas empresas mantengan la actividad y se adapten a la nueva demanda creciente de practicantes en contexto mixto de formación y práctica.

Nuestra investigación se plantea los siguientes interrogantes: ¿Qué dimensiones de la competencia emprendedora se han implementado durante la 
pandemia en el campo del ocio dentro de las escuelas de danza y actividades rítmicas y bailadas? ¿Qué dimensiones son las que definen a un emprendedor de éxito durante la pandemia? ¿Existe un modelo competencial emprendedor de referencia que permita superar crisis complejas?

Para guiar la investigación en la solución de los interrogantes planteados se formulan las siguientes hipótesis de trabajo:

- Hp 1: El emprendedor ha hecho uso de la aptitud de comunicación, la habilidad de sensibilizarse hacia los demás y su valor de compromiso social para mantener la danza como actividad de ocio enfocado a la salud en un momento de cierre de todas las empresas no esenciales.

- Hp 2: La actitud y la aptitud como componentes del emprendedor han permitido la adaptación al nuevo contexto para mantener la ocupación empresarial a través de la capacidad de resolución de problemas, la innovación, la iniciativa y la planificación.

- Hp 3: La capacidad para afrontar problemas y buscar soluciones ha adquirido relevancia para hacer frente a un incremento de demanda de la actividad unido a la incertidumbre por las continuas limitaciones.

\section{Metodología}

Para contrastar las hipótesis planteadas, se centra en un estudio cualitativo contextualizado en las escuelas de danza desde el inicio de la pandemia. Para el análisis de los datos, se centra en la teoría fundamentada, cuyo propósito es descubrir y desarrollar la teoría que se desprende del contexto investigado (Strauss y Corbin, 2002; San Martín, 2014; Luis, De la Torre y Jiménez, 2017).

La codificación teórica es el principal procedimiento de análisis para construir teoría, distinguiéndose tres procesos en la codificación: codificación abierta, codificación axial y codificación selectiva (Mendoza et al., 2020). Siguiendo la descripción planteada por San Martín (2014), en relación con la estructura seguida para la codificación, se aborda la codificación abierta con el fin de despojar conceptos e ideas que generen las categorías, y se analizan los datos para identificar y conceptualizar los significados que contienen las entrevistas. A partir de la codificación axial se identifican las frecuencias y sus relaciones 
entre las categorías obtenidas en la codificación abierta para dar cuenta de las teorías que subyacen tras el fenómeno. Con el propósito de obtener una categoría central con mayor nivel de abstracción y que exprese la competencia emprendedora implementada e integre las categorías de codificación abierta y axial, se ha realizado la codificación selectiva (Strauss y Corbin, 2002; San Martín, 2014).

Se ha llevado a cabo un proceso de categorización y análisis de datos cualitativo ejercido por el software ATLAS-ti versión Cloud, facilitando un análisis de frecuencias en relación con las familias de códigos y su densidad en los documentos analizados.

Como técnica de obtención de datos se realiza una entrevista de tipo semiestructurada. Este tipo de entrevista se caracteriza por ser simple y comprensible y estar abierta a los informantes sin depender de las categorías preestablecidas (Mendoza, Flores, Soledad, Cejas y Navarro, 2020).

La organización de las preguntas se ha establecido en torno a los siguientes bloques de contenido: aspectos sociodemográficos; aspectos empresariales; espíritu empresarial; espíritu emprendedor; competencias personales y atributos, $\mathrm{y}$, por último, conocimientos y aspectos vinculados a la actividad como práctica de ocio.

\subsection{Selección de la muestra}

Para la selección de la muestra, en primer lugar, desde el inicio de la pandemia se realiza un seguimiento en la red social Facebook de las escuelas formativas de danza españolas del sector privado que han mantenido la actividad en esta red desde el inicio de la pandemia hasta el final del estado de alarma. En segundo lugar, a través del buscador de Google se averigua el contacto de estas escuelas y se inicia una primera comunicación con las personas responsables; se realiza un muestreo por conveniencia según el criterio de "escuelas que han mantenido la empresa desde el inicio de la pandemia hasta la fecha de realización de la entrevista en el mes de marzo de 2021". Siguiendo el criterio de inclusión "empresa/escuela fundada antes del inicio de la pandemia", se seleccionan a partir de un muestreo aleatorio escuelas que tengan $<10$ años; 
de 10 a 20 años; de 20 a 30 años, y > 30 años. Por último, se excluyen las escuelas que no realizan la entrevista. El estudio finaliza con una muestra de 12 empresarios al frente de escuelas de danza que realizarán la entrevista. Los datos se analizan de forma sistemática y son categorizados con el objeto de hallar las relaciones entre los diferentes conceptos.

\section{ANÁLISIS DE RESULtAdos}

A partir de la documentación teórica y del contenido de las entrevistas realizadas, y con relación a las hipótesis planteadas en la investigación, se han identificado diferentes categorías en la codificación abierta, realizada mediante un proceso repetitivo entre la conceptualización de los datos y la elaboración de categorías y subcategorías, reconociendo las relaciones primarias entre dichos conceptos. En la tabla 1 se muestran las principales categorías que se corresponden con las dimensiones de la competencia emprendedora, así como subdimensiones de estas como subcategorías de análisis.

TABLA 1

Categorias y subcategorías de análisis

\begin{tabular}{|l|l|}
\hline \multicolumn{1}{|c|}{$\begin{array}{c}\text { CATEGORÍAS } \\
\text { DIMENSIONES }\end{array}$} & \multicolumn{1}{c|}{$\begin{array}{c}\text { SUBCATEGORÍAS } \\
\text { SUBDIMENSIONES }\end{array}$} \\
\hline \multirow{5}{*}{ ACTITUD } & Tolerancia/asunción de riesgos \\
\cline { 2 - 2 } & Autoestima, confianza en uno mismo \\
\cline { 2 - 2 } & Control percibido interno \\
\cline { 2 - 2 } & Necesidad de logro \\
\cline { 2 - 2 } & Orientado a la innovación \\
\cline { 2 - 2 } & Planificación \\
\cline { 2 - 2 } & Iniciativa \\
\cline { 2 - 2 } & Identificar oportunidades \\
\hline
\end{tabular}




\begin{tabular}{|c|c|}
\hline $\begin{array}{l}\text { CATEGORÍAS } \\
\text { DIMENSIONES }\end{array}$ & $\begin{array}{l}\text { SUBCATEGORÍAS } \\
\text { SUBDIMENSIONES }\end{array}$ \\
\hline \multirow{11}{*}{ APTITUD } & Capacidad de enfrentarse a problemas \\
\hline & Pensamiento crítico \\
\hline & Asumir responsabilidades \\
\hline & Autonomía \\
\hline & Capacidad de comunicación \\
\hline & Mejora del aprendizaje y de las actuaciones personales \\
\hline & Liderazgo \\
\hline & Trabajo en equipo \\
\hline & Tomar decisiones \\
\hline & $\begin{array}{l}\text { Utilización de las tecnologías de la información y la comuni- } \\
\text { cación }\end{array}$ \\
\hline & Capacidad de negociación (persuasión) \\
\hline \multirow{4}{*}{ CONOCIMIENTOS } & Formación en el área \\
\hline & Otra formación \\
\hline & Experiencia laboral \\
\hline & Formación para la adaptación a la situación \\
\hline \multirow{3}{*}{ HABILIDADES } & Creatividad \\
\hline & Relaciones sociales \\
\hline & Sensibilidad \\
\hline VALORES & Compromiso personal \\
\hline \multirow{4}{*}{$\begin{array}{l}\text { OTRAS COMPETENCIAS ASOCIA- } \\
\text { DAS AL EMPRENDIMIENTO }\end{array}$} & Tolerancia al fracaso \\
\hline & Actitud mental positiva \\
\hline & Gestión de recursos (optimización) \\
\hline & Inteligencia emocional \\
\hline
\end{tabular}

Fuente: elaboración propia 
El análisis de frecuencias por grupos de categorías proporciona una visión general de las dimensiones movilizadas para hacer frente a la situación de crisis desde el inicio de la pandemia. En la figura 1 se puede ver esta relación.

FIGURA 1

Frecuencias y porcentajes de citas por grupos de categorías

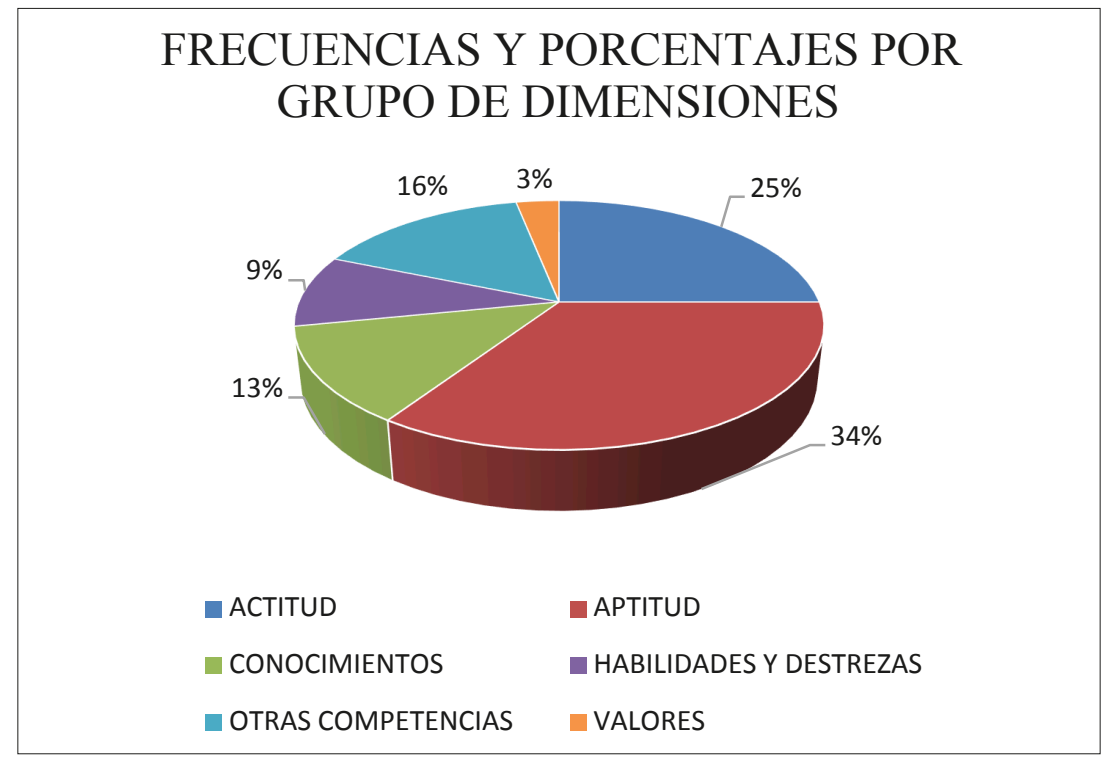

Fuente: elaboración propia

De forma general, y antes de analizar los resultados de cada dimensión, la capacidad implementada en mayor grado ha sido la aptitud, lo que refleja la importancia que tienen los rasgos innatos e intrínsecos de la persona. En segundo término, la actitud y los rasgos que la definen. En tercer lugar, se han implementado como competencias relacionadas con el emprendedor la tolerancia al fracaso, una actitud mental positiva, la optimización de recursos y la inteligencia emocional (Aragón y Baixauli, 2010; Enciso-Congote, 2010; De la Torre et al., 2019). Por último y con una implicación similar, las dimensiones relacionadas con los conocimientos, las habilidades y destrezas y los valores.

Si se analizan los rasgos que definen cada dimensión, considerando la aptitud como las más implementada, destaca la capacidad de comunicación como 
el rasgo que define al emprendedor en las relaciones con los demás, ya que da importancia a la adaptación en la situación de crisis. Mantener la comunicación constante con los usuarios ha sido el rasgo de más porcentaje, seguido del pensamiento crítico y la capacidad de encontrar soluciones a los problemas. La acción de visibilizar su negocio a partir de un aprendizaje, la optimización e incremento en el uso de las tecnologías de la información y la comunicación son los siguientes rasgos con más frecuencia, y han fomentado que los practicantes de la actividad se sensibilizaran con el sector y la adhesión de nueva clientela. En la figura 2 puede verse la relación de los rasgos de la aptitud y su implementación en relación con la frecuencia y su porcentaje.

FIGURA 2

Frecuencia y porcentaje de los rasgos que conforman la aptitud

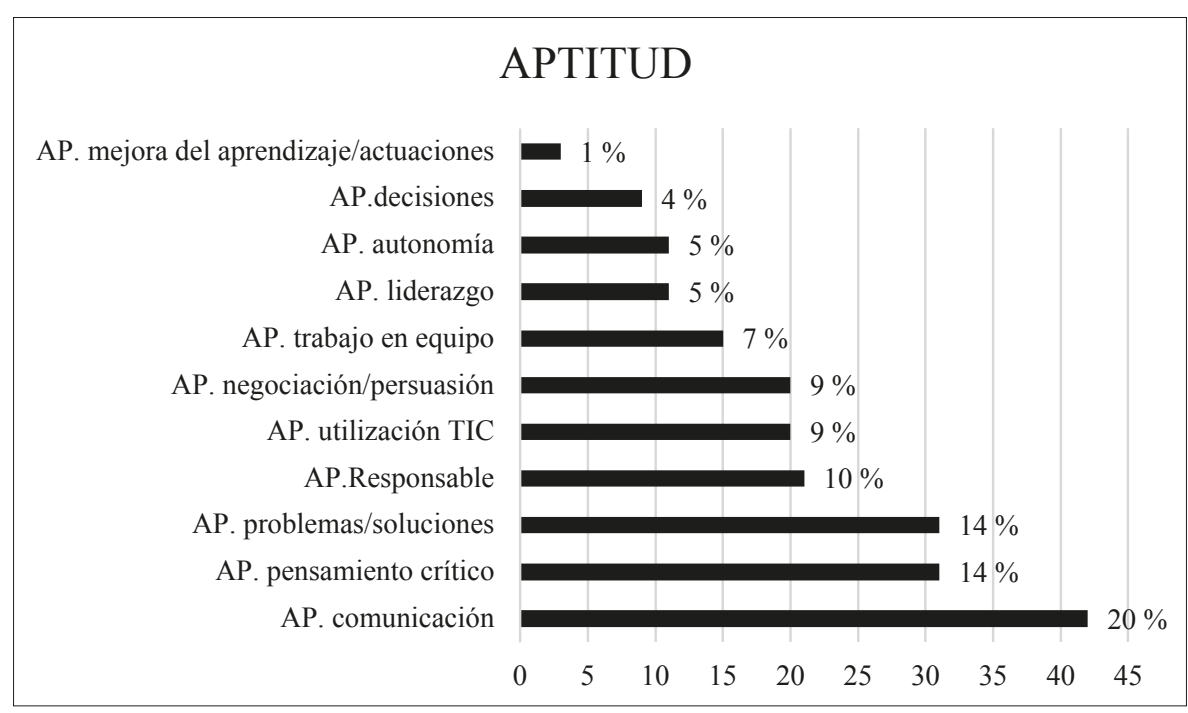

Fuente: elaboración propia

Los resultados muestran cómo, en segundo grado de importancia, la actitud del emprendedor ha permitido, a través de la capacidad de innovación y la iniciativa como rasgos fundamentales, identificar nuevas oportunidades para redefinir su servicio y poder adaptarse, a partir de la capacidad de planificación a los diferentes contextos que han sucedido desde el inicio de la pandemia. 
La disposición a generar cosas diferentes y originales, de no estancarse ni paralizar la empresa, no solo para solucionar el problema del momento, sino llevados por la iniciativa de indagar nuevos enfoques y formas de atender a la situación de la actividad, unido a la actitud de planificación para poder llevar a cabo todos estos cambios necesarios con autoconfianza, ha permitido progresar y sobrevivir, siendo la innovación la dimensión que se ha mostrado en mayor porcentaje. La figura 3 muestra la relación entre los componentes de la actitud como la conducta permanente de administrar los recursos y así lograr un servicio adaptado.

FIGURA 3

Frecuencia y porcentaje de los rasgos que conforman la actitud

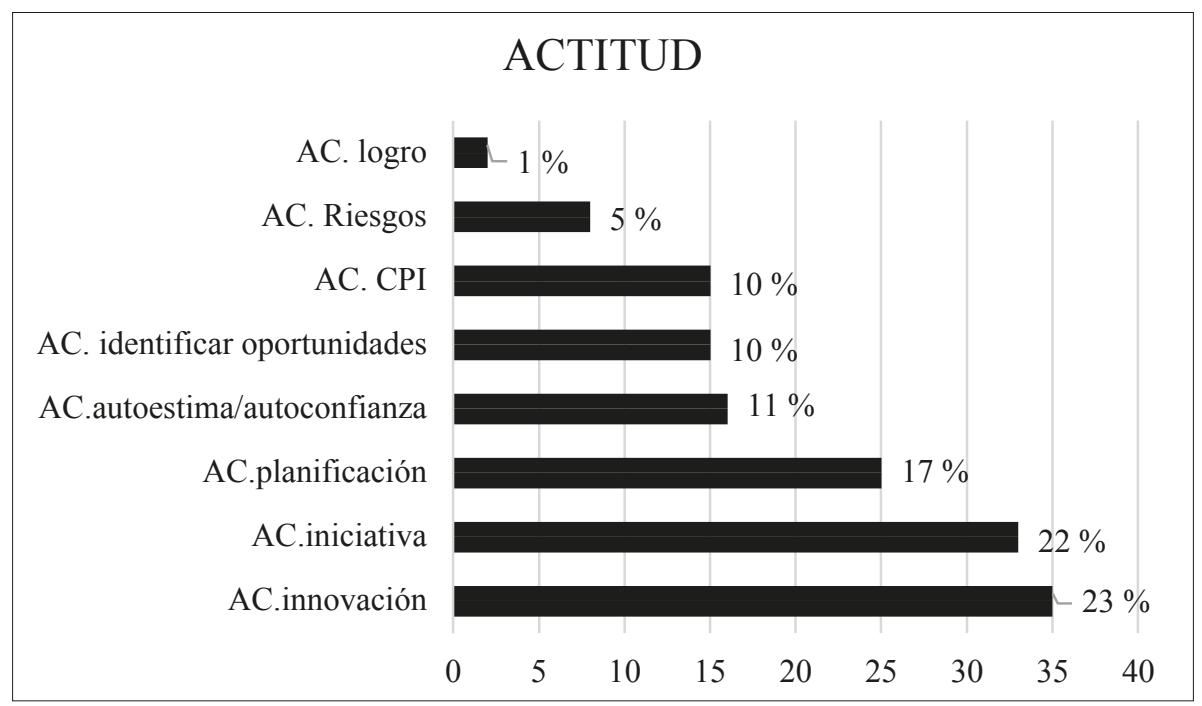

Fuente: elaboración propia

Y, afrontando el último grado de desempeño dentro de las dimensiones implementadas como rasgos definitorios del emprendedor, se desvelan los conocimientos, habilidades y destrezas y valores que han sido activados para la adaptación. En este ámbito, la sensibilidad ha sido el aspecto más movilizado, seguido del compromiso personal. En este caso, una sensibilidad hacia las necesidades de toda la población, que dirige a estos emprendedores a la 
acción por un compromiso particular y social como valor, se complementa con el aprendizaje y la formación para la transformación y la utilización de las tecnologías de la informática y la comunicación mediante la innovación y creatividad. Se han aplicado tecnologías ya conocidas, pero nunca utilizadas, con el objetivo de ofrecer el servicio en línea con una demanda masiva, y, gracias a la creatividad como aspecto que se asocia a la actitud innovadora, se ha modificado por completo la actividad para convertirla en un nuevo servicio adaptado a las necesidades de la población. La figura 4 muestra la relación entre los componentes de estas dimensiones y su implementación.

FIGURA 4

Frecuencia y porcentaje de los rasgos que conforman el conocimiento, habilidades y destrezas y los valores

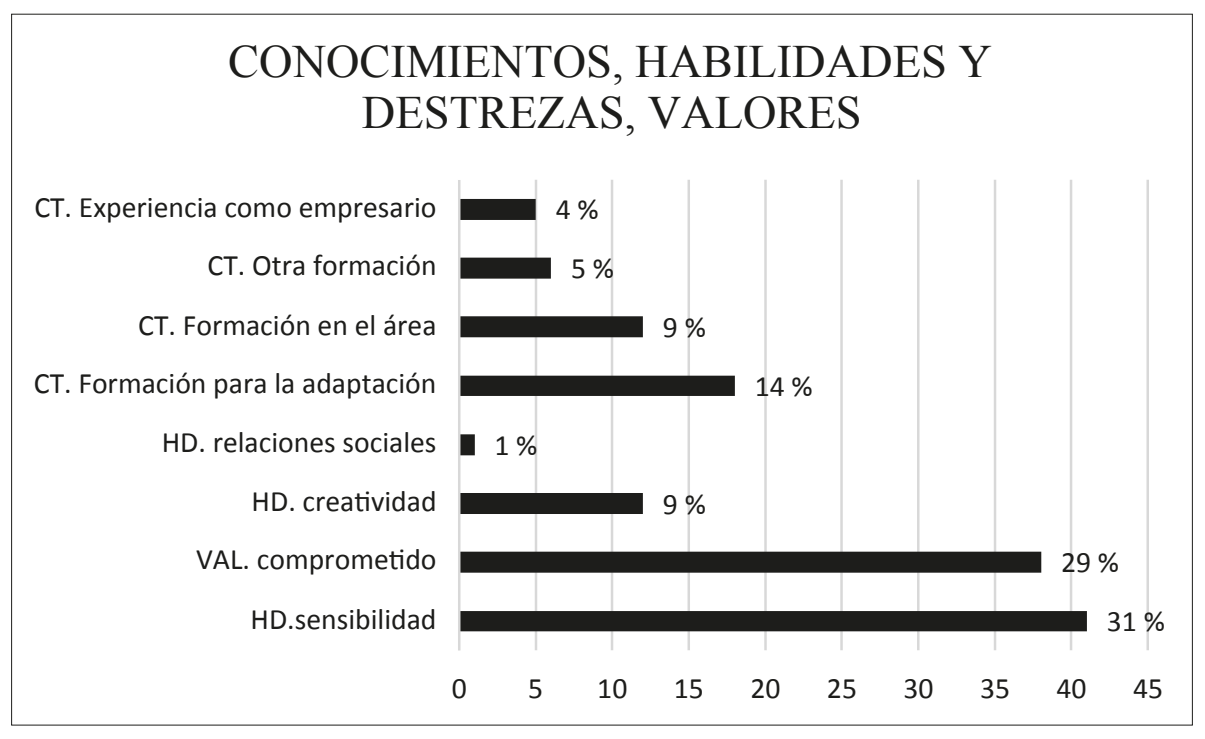

Fuente: elaboración propia

Considerando todas las dimensiones analizadas, los resultados confirman la capacidad de enfrentarse y manejar los problemas y resolverlos como el rasgo más sobresaliente, seguido por la capacidad de comunicación y relación con los demás, la sensibilidad hacia la situación de la población y el compromiso personal con la sociedad. Unidos al rasgo de innovación e iniciativa, han com- 
portado una redefinición del servicio prestado al contexto actual de demanda de la actividad.

El emprendedor no ha interpretado la adaptación del nuevo servicio como un riesgo, sino como una responsabilidad social, y con pensamiento crítico ha tomado la situación como una oportunidad para mantener su actividad empresarial por medio de la innovación y la iniciativa. La figura 1 muestra la nube de palabras en la que se pueden identificar los rasgos implementados en función de su importancia para la adaptación.

Nube de palabras en función de la importancia de subdimensiones y su implementación

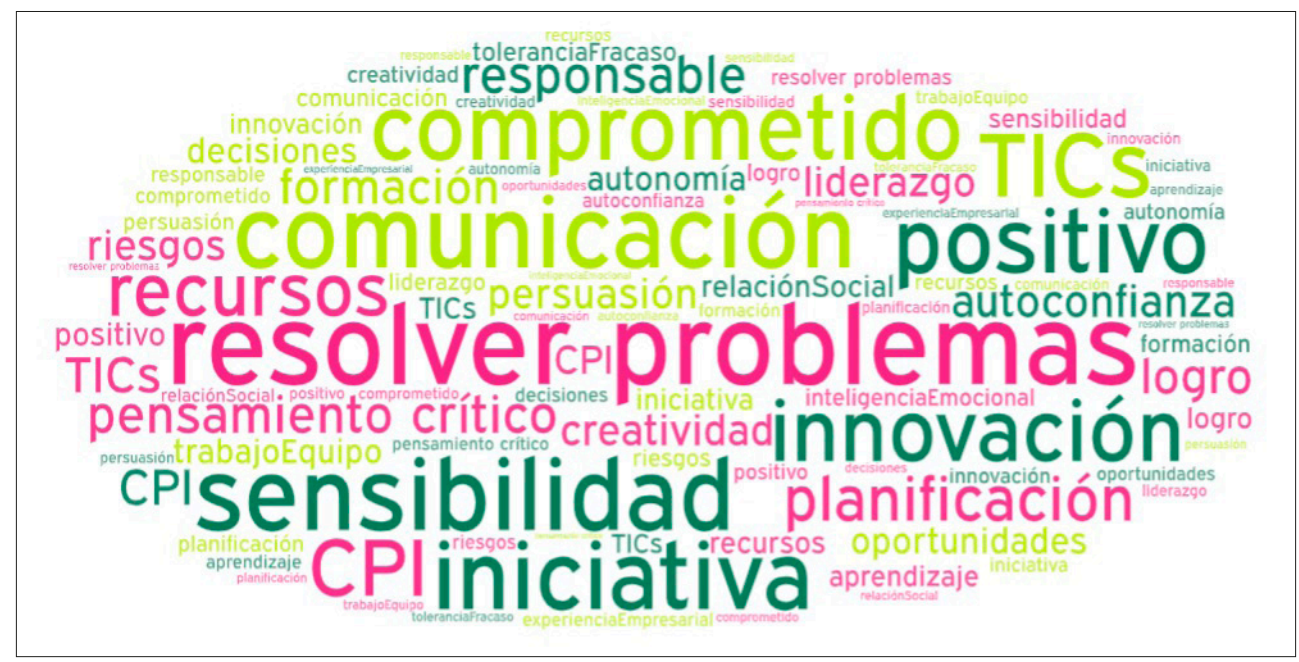

Fuente: elaboración propia

\section{Discusión Y CONCLUSIONES}

La paralización inicial de las actividades no consideradas esenciales y la incertidumbre de la situación han sido los aspectos que más han preocupado a los emprendedores y empresarios. La investigación muestra cómo algunas escuelas de danza de España han mantenido su empresa y se han adaptado para sobrevivir (Fuentes-Fuentes y Neira-Gómez, 2021), gracias a la disposición 
de un conjunto de habilidades vinculadas con la competencia emprendedora y las dimensiones que subyacen tras estas habilidades (Alda-Varas, VillardónGallego y Elexpuru-Albizuri, 2012).

Desde el inicio, y todavía en la actualidad, esta situación problemática está repleta de incertidumbres derivadas de la propia dinámica de obligaciones en la vida de la población provocada por las variaciones de la pandemia. El emprendedor ha comprendido y afrontado esta problemática con acciones que rebasan las referidas a tareas y habilidades de ejecución profesional (Adler, 2013; Coll, 2014; De la Torre et al., 2019).

A partir de la actitud como dimensión, el sector de la danza ha identificado la necesidad demandada por la población, en relación con estas actividades, como una ocasión y una posibilidad para la renovación (Morán, 2020; Nyenhuis, Greiwe, Zeiger, Nanda y Cooke, 2020), transformando la realidad en una oportunidad (Ferreiro, 2014).

Los resultados muestran que la aptitud, como aspecto con carácter permanente e intrínseco a la persona, ha sido el rasgo más implementado a través de la capacidad de enfrentarse a los problemas con la responsabilidad de encontrar la solución (Jiménez-Zarco, 2012). Además, esta aptitud se ha visto reforzada por la creatividad como habilidad necesaria para el manejo de problemas, lo que ha permitido desarrollar nuevas estrategias y transformar el servicio de forma dinámica, según han ido surgiendo las dificultades (Peñaherrera y Cobos, 2012). A todas estas personas al frente de escuelas de danza les ha caracterizado la habilidad para la comunicación con la población que accedía a su actividad y la gestión eficaz en las relaciones sociales como aspectos con impacto en el rendimiento de su empresa tras el estado de alarma (Gerli, Gubitta y Tognazzo, 2011).

Tal y como apuntan Cantón, García y Iuit (2014), los emprendedores han encontrado la motivación para la resolución de problemas en el compromiso social, como uno de los valores que ha suscitado sus acciones, y han dirigido este compromiso al desarrollo social y a la demanda generada por el problema de la pandemia (González y López, 2012). Así, llevados por la sensibilidad como una de sus habilidades, dentro de sus rasgos principales, se ha priorizado su responsabilidad social a partir de su compromiso con la situación mundial (Jiménez-Zarco, 2012); y al igual que otros sectores profesionales no considerados esenciales han adaptado su producto (Haeffele, Hobson y Storr, 
2020), enfocando sus negocios con objetivos diferentes, se ha comprobado cómo el sector de la danza se ha movilizado por otros aspectos más allá de lo económico. Así, se han desarrollado iniciativas sociales (Jiménez-Zarco, 2012) que han fomentado la adhesión, el interés y la predisposición de la población hacia la práctica de estas actividades, buscando diferentes objetivos (Capasso et al., 2020; Dalmases, 2020; Encalada, 2020; Hansen, Main y Hartling, 2021; Lara-Aparicio, Mayorga-Vega y López-Fernández 2021; Trevino et al., 2021).

Una de las capacidades que ha permitido la continuación de la actividad, como bien social durante el estado de alarma y continuando como actividad empresarial tras el confinamiento, ha sido la orientación hacia la innovación y la iniciativa empresarial (Hisrich, Peters y Sheperd, 2005; Duarte, 2009; Ferreiro, 2014; De la Torre et al., 2019). Utilizando las TIC y tecnologías ya conocidas con anterioridad, han desarrollado la actividad en formato en línea y mixto en función de las necesidades demandadas en cada momento y por cada usuario para beneficiarse de la oportunidad (Gerli, Gubitta y Tognazzo, 2011). Se ha creado un servicio renovado con el elemento original de la realización de la actividad desde cada hogar, un nuevo contexto de práctica en línea o mixta que ha podido realizarse gracias a la formación de los profesionales que han adaptado la metodología para la práctica en los distintos contextos (Ferreiro, 2014; De la Torre et al., 2019). Esta habilidad de iniciativa e innovación ha permitido la continuación de la actividad en todo momento, generando un sentimiento de satisfacción y demanda en los consumidores (Peñaherrera y Cobos, 2012), al poder realizar una actividad beneficiosa para la salud y que se ha adaptado a las necesidades individuales (Alpert, 2011; Andreu-Cabrera, 2020; Hammami et al., 2020; Bohn y Hogue, 2021; Hansen, Main y Hartling, 2021; Lara-Aparicio, Mayorga-Vega y López-Fernández, 2021; Re, 2021; Trevino et al., 2021).

Así, los emprendedores del sector han actuado guiados por diferentes rasgos: a partir de su sensibilidad personal y su compromiso social han ofrecido su actividad apoyada en su estructura empresarial como bien necesario del momento (Alcaraz, 2011; Gerli, Gubitta y Tognazzo, 2011; Haeffele, Hobson y Storr, 2020); apoyándose en su capacidad de comunicación con los demás y en su pensamiento crítico han identificado la necesidad social y la demanda como un oportunidad (Nyenhuis et al., 2020); han orientado su capacidad innovadora en iniciativas sólidas, lo que ha dado lugar a un nuevo mercado 
nunca planteado, y han convertido las ideas surgidas por esta necesidad social en acciones a partir de la innovación, la iniciativa y la creatividad (De la Torre et al., 2019).

Llevados por la sensibilidad y el compromiso social, han hecho uso de su competencia emprendedora, aportando un servicio renovado, superando anomalías y cambiando la vida de la gente con su producto (De la Torre et al., 2019); han demostrado con su rapidez y adaptación poseer creatividad, ser innovadores y capaces de reorganizar su empresa, y de asumir riesgos (Malagón y Colombia, 2003). Este espíritu y su iniciativa empresarial organizacional han permitido a la dirección de las escuelas de danza buscar oportunidades, utilizando los recursos que ya poseían y adquiriendo nuevos conocimientos para el uso de los recursos tecnológicos necesarios (Yahou, 2020), añadiendo importancia al desarrollo del espíritu empresarial como componente clave del desarrollo social y económico, debido a su potencial para la innovación y la competitividad (Luis-Rico et al., 2020a). No se puede pensar que el espíritu empresarial sea la única solución para los problemas económicos actuales, pero se ha identificado como uno de los componentes clave para el crecimiento y el desarrollo social y económico en las situaciones de crisis (Luis-Rico et al., 2020b).

Adicionalmente, a la vista de la situación actual y teniendo en cuenta que el empresario español ha visto oportunidades en los momentos de crisis como la que todavía se está viviendo actualmente, en la que continúan modificando y diversificando su modelo de negocio (Fuentes-Fuentes y Neira-Gómez, 2021), se concluye plantear la importancia de promover la investigación en la figura del emprendedor y el emprendimiento como recurso de empuje para afrontar períodos de crisis como la vivida provocada por la COVID-19 (Nicolás y Rubio, 2020). Esta consideración implica una nueva conceptualización, donde la educación en la competencia emprendedora debe atender a todos los rasgos que se han visto implementados y que desenvuelven al emprendedor en los planos intrapersonal, interpersonal, profesional y social (Luis, De la Torre y Jiménez, 2017).

Por otro lado, la investigación en sectores como el estudiado se cree que contribuye a la información necesaria respecto a las necesidades de la actividad y los centros donde se realizan, y así promover la red empresarial del país, lo que ha provocado una mayor velocidad en la regeneración económica. 
Como una de las estrategias para la recuperación del sector, se propone dar a los empresarios el espacio necesario para crear soluciones específicas a los problemas que van surgiendo, considerando la experiencia en desastres pasados que han demostrado que son los emprendedores quienes han movilizado sus competencias y se han adaptado a las circunstancias y necesidades cambiantes para mantener las empresas, teniendo en cuenta las realidades de cada una.

No se puede obviar una referencia final a las limitaciones del estudio, que se erige como un primer paso para una investigación ampliada, que implique a escuelas por comunidades y provincias, incrementando la envergadura del estudio.

\section{BiBLIOGRAFÍA}

Adler, A. C. (2013). Competencias, Rasgos, Principios y Reglas de la ética profesional. Revista Xihmai 3(6), 1-16. Recuperado de: https://dialnet.unirioja.es/servlet/articulo?codigo $=4953791$

Alcaraz, R. (2011). El emprendedor de éxito (4. ${ }^{\mathrm{a}}$ ed.). D. F. México: McGrawHill/Interamericana editores. (consulta: 20121). ISBN: 978-607-15-0611-5.

Alda-VARAS, R., Villardón, L. y EleXPURU, I. (2012). Propuesta y validación de un perfil de competencias de la persona emprendedora. Implicaciones para la formación. Educational Psycology 10(3), 1057-1080. Recuperado de: https://www.redalyc.org/pdf/2931/293124654006.pdf

Alpert, P. T. (2011). The health benefits of dance. (SAGE, Ed.) Home Health care management \& Practice 23(2), 155-157. DOI: https://doi. org/10.1177/1084822310384689.

Andreu-CABrera, E. (2020). Actividad física y efectos psicológicos del confinamiento por COVID-19. INFAD Revista de Psicología 2(1), 209-220. Recuperado de: https://revista.infad.eu/index.php/IJODAEP/article/view/1828. ISSN: 0214-9877.

Aragón, A. y BaIXAuli, J. S. (2010). El reto de aprender: factores clave. Navarra: Aranzadi. ISBN: 978-84-470-3353-9.

Bohn, J. y Hogue, S. (2021). Changing the game: college dance training for well-being and resilience amidst the COVID-19 crisis. Health promotion practice 22(2), 163-166. DOI: https://doi.org/10.1177/1524839920963703. 
Burzynska, A. Z., TAylor, B. K. y Knecht, A. M. (2017). The dancing brain: structural end functional signatures of expert dance training. HUman Neuroscience 11(566), 20. DOI: https://doi.org/10.3389/fnhum.2017.00566.

Cantón, L. C., García, A. y Iuit, M. C. (2014). El enfoque de competencias para formar emprendedores: Evaluación del modelo de la Universidad Autónoma de Yucatán, México. TEC. Empresarial 8(2), 29-40. Recuperado de: https://revistas.tec.ac.cr/index.php/tec_empresarial/article/view/1987

Capasso, V., Camezzana, D., Sabrina Mora, A. y Sáez, M. (2020). Las artes escénicas en el contexto del ASPO. Demandas, iniciativas, políticas y horizontes en la danza y el teatro. Questión/Periodismo/Comunicación 2(66), 1-19. DOI: https://doi.org/10.24215/16696581e470

Capel, J., Cruvinel, V., Mendoça, M. y Celeno, C. (2018). Quality of life of women who practice dance: a systematic review protocol. Systematic Reviews 7(92), 1-6. DOI: https://doi.org/10.1186/s13643-018-0750-5.

Coll, C. (2014). La evaluación continuada como instrumento para el ajuste de la ayuda pedagógica y la enseñanza de competencias de autoregulación. Cuaderno de Pedagogía Universitaria 8(15), 14-20. Recuperado de: https:// acortar.link/pCDkgv

DALmases, I. (29 de abril de 2020). Bailando en línea y en directo para ponerse en forma en el confinamiento. ElPeriódico. Recuperado de: https://acortar. link/Zd6BgQ (consulta: 29/04/2020).

Damas, A. (2012). Promover el emprendimiento en secundaria. Aula de innovación educativa 213-214, 38-44.

De la Torre, T., Luis, M. I., Palmero, C., Escolar, M. C. y Jiménez, A. (2019). Educación y competencia emprendedora. Retos teóricos e implicaciones socio pedagógicas. Madrid: Dykinson. ISBN: 978-84-1324-444-0.

Ding, Y., Guo, C., Yu, S., Zhang, P., Feng, Z., Sun, J., ..., Zhuang, H. (2021). The effect of dance-based mind-motor activities on the quality of life in the patinets recovering from COVID-19. A protocol for systematic review and meta-analysis. Medicine 11(100), 1-5. DOI: http://dx.doi.org/10.1097/ MD.0000000000025102.

DuArte, J. E. (2009). Emprendedor: crear su propia empresa. Starbool, Paracuellos de Jarama. ISBN: 978-84-92-65000-2.

ENCALADA, E. (25 de abril de 2020). La práctica de danza es una aliada durante la pandemia. El Comercio. Recuperado de: https://acortar.link/8UhrGV 
Enciso-Congote, J. D. (2010). El emprendimiento y el bien común: competencias complemetarias o excluyentes. Educación y Educadores 13(1), 63-76. Recuperado de: https://dialnet.unirioja.es/servlet/articulo?codigo $=3256384$

Espínola, V. y Torres, L. A. (2020). Análisis cualitativo de modelos de negocio para el emprendimiento social. Entreciencias: Diálogos en la Sociedad del Conocimiento 22, 1-16. DOI: https://doi.org/10.22201/ enes1.20078064e.2020.22.75431.

FANCOURT, D. y FInN, S. (2019). What is the evidence on the role of the arts in improving health amd well-being? A scoping review. World Health Organization. OMS. Copenhagen: WHO regional office for Europe. Recuperado de: https://www.ncbi.nlm.nih.gov/books/NBK553773/. ISBN: 978-92-8905455-3.

FERreiro, F. (2014). Factores que contribuyen a la existencia del emprendedor. Revista criterios de Res pública Fulget 13. Recuperado de: https://www. researchgate.net/publication/322794974_Factores_que_contribuyen_a_la_ existencia_del_emprendedor. (consulta: 2021).

Fuentes-Fuentes, M. D. y Neira, I. (2021). Situación del emprendimiento en España ante la crisis del COVID-19. Análisis y recomendaciones. Red GEM. Observatorio del Emprendimiento de España. Santander: Observatorio del Emprendimiento de España. Recuperado de: https://acortar.link/ MpTycE. ISBN: 978-84-09-20395-6.

García-Parada Arias, G. y AguAdo, J. (2020). Emergencia y emprendimiento social. Ahora sí es el momento. Emprender contra el COVID-19. Noticias CIELO 8. Recuperado de: https://acortar.link/HCQOEI. ISSN-e 2532-1226. Gerli, F., Gubitta, P. y Tognazzo, A. (2011). Entrepreneurial competencies and firm performance: an empirical study. VIII International Workshop on Human Resource Management. Sevilla. Recuperado de: https://www. researchgate.net/publication/228314296_Entrepreneurial_Competencies and_Firm_Performance_An_Empirical_Study

GonzÁlez, J. A. y LóPez, C. D. (2012). El emprendimiento en los sistemas universitarios (vol. 4). (CAF, Ed.) Monterrey: Cyngular. Recuperado de: https://scioteca.caf.com/handle/123456789/370?show=full. ISBN: 978980-6810-74-7. 
Haeffele, S., Hobson, A. y Storr, V. (2020). Coming back from COVID-19: Lessons in entrepreneurship from Disaster Recovery Research. SSRNElectronic Journal. DOI: https://doi.org/10.2139/ssrn.3592966.

Hammami, A., Harrabi, B., Mohr, M. y Krustrup, P. (2020). Physical activity and coronavirus disease 2019 (COVID-19): specific recommendations for home-based physical training. Managing Sports and Leisure, 1-6. DOI: https://doi.org/10.1080/23750472.2020.1757494.

Hansen, P., Main, C. y Hartling, L. (2021). Dance intervention affects social connections and body appreciation among older adults in the long term despite COVID-19 social isolation: a mixed methods pilot study. Frontiers in psychology 12, 1-17. DOI: https://doi.org/10.3389/fpsyg.2021.635938.

Hisrich, R., Peters, M. y ShePerd, D. (2005). Entrepreneurship (6. a ed.). Madrid: McGraw-Hill/Interamericana de España. ISBN: 978-84-48-19839-8.

JARAmillo, B. (2015). Emprender e innovar en época de crisis. Revista económica y negocios, 18-20. Recuperado de: www.researchgate.net/publication/281284997.

JimÉneZ-ZARCo, A. I. (2012). Emprender en tipos de crisis: el emprendimiento innovador como base de generación de valor. VIII Congreso virtual. Emprendimiento, Pymes y gestión del conocimiento 72-50. Bogotá: Universidad Santo Tomás (consulta: 2021).

Lakes, K. D., Marvin, S., Rowley, J., San Nicolás, M., Arastoo, S., Viray, L., ... JURNAK, F. (2016). Dancer perceptions of the cognitive, social, emotional, and physical beneficts of modern styles of partnered dancing. Complementary Therapies in Medicine 26, 117-122. DOI: https://doi.org/10.1016/j. ctim.2016.03.007

Lara-Aparicio, M., Mayorga-Vega, D. y López-Fernández, I. (2021). Expressive movement $\&$ creative dance practice in times of quarantine: The \#VIDLOP movement. Movimiento. Revista de educaçao física da UFRGS 27, 1-19. DOI: https://doi.org/10.22456/1982-8918.105802.

Luis, M. I., De la Torre, T. y JimÉNez, A. (2017). Emprendimiento y ocio en la formación inicial del profesorado de la Educación Primaria. OBETS. Revista de Ciencias Sociales 12(1), 121-149. DOI: https://doi.org/10.14198/ OBETS2017.12.1.15.

Luis-Rico, I., Escolar-Llamazares, M. C., De la Torre-Cruz, T., Jiménez, A., Herrero, A., Palmero-Cámara, C. y Jiménez-Eguizábal, A. (2020a). 
Entrepreneurial Interest and Entrepreneurial Competence among Spanish Youth: An analysis with Artificial neural networks. Sustainability 12(35), 1-17. DOI: https://doi.org/10.3390/su12041351.

Luis-Rico, M. I., Escolar-Llamazares, M. C., de la Torre-Cruz, T., Herrero, A., Jiménez, A., Palmero-Cámara, C. y Jiménez-Eguizábal, A. (2020b). The association of parental Interest in Entrepreneurship with the entrepreneurial interest of spanish youth. International Journal of Environmental Research and Public Health 17(4744), 1-17. DOI: https://doi.org/10.3390/ ijerph17134744.

Malagón, F. A. y Colombia, D. C. (2003). El espíritu emprendedor y la creación de empresa. Colombia: FED. Recuperado de: https://acortar.link/6QHpZV MÁrquez, A. J. (2020). Inactividad física, ejercicio y pandemia COVID-19. (I. u. deporte, Ed.). VIREF Revista de educación física 9(2). Recuperado de: https://revistas.udea.edu.co/index.php/viref/article/view/342196. ISSN: 2322-9411.

Mendoza, D. J., Flores, E. M., Soledad, L., Cejas, M. F. y Navarro, M. (2020). Práctica pedagógica de la educación ecuatoriana en el siglo XXI. Edetania 57, 111-141. DOI: https://doi.org/10.46583/edetania_2020.57.437.

MorÁn, R. C. (2020). Emprendimiento global: una visión en tiempos del Covid-19. Revista Venezolana de gerencia 92, 1288-1295. Recuperado de: https://hdl.handle.net/11537/26839. ISSN: 2477-9423.

Nicolás, C. y Rubio, A. (2020). Emprendimiento en épocas de crisis: Un análisis exploratorio de los efectos de la COVID-19. Small Business International Review 4(2), 53-66. DOI: https://doi.org/10.26784/sbir.v4i2.279.

Nyenhuis, S. M., Greiwe, J., Zeiger, J. S., Nanda, A. y Cooke, A. (2020). Exercise and Fitness in the age of social distancing during the COVID-19 Pandemic. The Journal of Allergy and clinical immunology. In practice 8(7), 2152-2155. DOI: https://doi.org/10.1016/j.jaip.2020.04.039.

Peñaherrera, M. y Cobos, F. (2012). La creatividad y el emprendimiento. REICE. Revista iberoamericana sobre calidad, eficacia y cambio en educación 10(2), 239-247. Recuperado de: https://www.redalyc.org/ pdf/551/55124596016.pdf. ISSN: 1696-4713.

RE, M. (2021). Isolated systems towards a dancing constellation: coping with the Covid-19 lockdown through a pilot dance movement therapy tele- 
intervention. Body, Movement and Dance in Psychotherapy 16, 9-18. DOI: https://doi.org/10.1080/17432979.2021.1879934.

Real Decreto 463/2020, de 14 de marzo, por el que se declara el estado de alarma para la gestión de la situación de crisis sanitaria ocasionada por el COVID-19. Boletín Oficial del Estado, 14 de marzo de 2020, n. ${ }^{\circ} 67$, pp. 25390 a 25400.

SAn Martín, D. (2014). Teoría fundamentada y atlas ti: recursos metodológicos para la investigación educativa. Revista electrónica de investigación educativa 16(1), 104-122. Recuperado de: http://redie.uabc.mx/vol16nol/ contenido-sanmartin.html.

SEPE, S. P. (2020). 2020. Informe del mercado de Trabajo Estatal. Datos 2019. Madrid: Ministerio de Trabajo y Economía Social. Recuperado de: http:// publicacionesoficiales.boe.es.

Social, S. G. (2021). Informe trimestral de análisis del mercado de trabajo. N. ${ }^{\circ}$ 136. Marzo 2021. Madrid: Ministerio de Trabajo y Economía Social. Recuperado de: https://acortar.link/kLYGzK

Strauss, A. y Corbin, J. (2002). Bases de la investigación cualitativa. Técnicas y procedimientos para la teoría fundamentada. Colombia: Universidad de Antioquía. Recuperado de: https://diversidadlocal.files.wordpress. com/2012/09/bases-investigacion-cualitativa.pdf. ISBN: 958-655-624-7.

Trevino, K., Raghunathan, N., Latte, S., Polubriaginof, F. C., Jensen, C., AtKinson, T. M., ..., MAO, J. J. (2021). Rapid deployment of virtual mindbody interventions during the COVID-19 outbreak: feasibility, acceptability, and implications for future care. Support Care Cancer 29, 543-546. DOI: https://doi.org/10.1007/s00520-020-05740-2.

Yaноu, H. (2020). Ambidexterity leadership, transformational, leadership, and corporate entrepreneurship. Evaluating the moderating role of behavioral and psychological factors. Revista Conrado 16(75), 150-158. ISSN: 1990-8644. Recuperado de: https://conrado.ucf.edu.cu/index.php/conrado/ article/view/1402 